\title{
La stabilité d'une chambre d'équilibre placée sur la galerie de fuite d'une usine
}

\author{
Extension, au système de fuite, des résultats obtenus \\ par M. André Gardel pour le système d'amenée. \\ Effet de l'énergie cinétique dans la galerie \\ The stability of a downstream surge tank
Extension of Mr. André Gardel's results for an upstream surge tank to one on the downstream side of a power station. \\ Effect of the kinetic energy on the base of the structure \\ PAR G. D. RANSFORD \\ B.C.E., B.A. (HONS.), INGËNIEUR-DOCTEUN \\ INGÉNRUT AU LABORATOIRE DAUPHINOIS D'HYDRAULIQUE (SOGREAH, GRENOBLE)
}

\begin{abstract}
Dans un livre récent, M. André GARDEL a tiré au clair l'effet de l'énergie cinétique a la base d'ane cheminée amont sin la stabilité de celleci. L'extension de sa méthode à une cheminée aval (cas d'une usine souterraine) conduit i ce résultat inattendu : l'énergie cinétique améliorerait la stabilité, au lien d'y porter atteinte, comme on l'a cru jusqu'ici. L'auteur explique pourquoi des essais sur modele réduit s'imposent chaque fois qu'on aura un ouvrage déterminé à étudier.
\end{abstract}

\begin{abstract}
In a recent book, Mr. André Gandel has clarified the effect of kinetic energy on an $n p$ stream surge tanl. The present application of his method to a downstream structure (case of an underground power plant) leads to the unexpected result that the linetic energy actually aids stability, instead of impairing it (as previously believed). The recessity of model tests for studying specific structure is explained.
\end{abstract}

\section{INTRODUCTION}

A l'occasion de l'étude de deux usines souterraines possédant chacune une galerie de fuite très courte munie d'une cheminée d'équilibre, il nous a paru d'un très grand intérêt d'entreprendre un examen approfondi des conditions de stabilité vis-à-vis des petites perturbations dues au réglage. Vu que, dans ce genre d'aménagement, la hauteur due à la vitesse, à la base de la cheminée, atteint facilement une valeur au moins voisine de celle des pertes de charge dans la galerie en: aval, la précision de notre estimation de la section critique de la chambre, pour laquelle lamortissement des petites oscillations n'est plus assuré, dépendra en grande partie de notre juste appréciation de l'effet de cette énergie cinétique. Selon les hypothèses qui leur servent de hase de départ, les techniciens les mieux avertis pourront faire des estimations, variant du simple au double, quant à la valeur de cette section critique.

On a eu depuis longtemps le sentiment que, dans le cas habituel d'une cheminée en amont, une fraction seulement de la hauteur due à la vitesse à la base de la cheminée devrait être 
ajoutée aux pertes de charge $P_{0}$ figurant dans le dénominateur de la formule classique de Thoma:

$$
\mathrm{F}_{t}=\frac{\mathrm{W}_{02}^{2}}{2 g} \quad \frac{\mathrm{LF}_{g}}{\mathrm{P}_{0} \mathbf{H}_{0}}
$$

qui à l'origine n'en tenait pas compte. M. A. GarDEL (*) a eu le grand mérite d'avoir tiré au clair la signification physique de la fraction dont il s'agit (environ $70 \%$ dans les cas courants), d'avoir rattaché cette grandeur aux caractéristiques de l'installation, et enfin d'avoir ouvert la voie à des compléments d'étude tels que la présente note.

En effet, les raisonnements simplistes qu'on a pu faire jusqu'ici au sujet de l'énergie cinétique dans les systemes de fuite munis de chambre d'équilibre, conduisaient à retrancher la totalité de l'énergie $\mathrm{E}_{0}$ en question des pertes de charge $P_{0}$. Dans une installation tout à fait concevable, où la hauteur due à la vitesse $\mathrm{E}_{0}$ égalerait, ou dépasserait, $\mathrm{P}_{0}$, il y aurait instabilité, quelle que soit la section $F$ de la chambre. Aucun effort d'imagination ne permeltait d'étayer ou d'infirmer cette supposition, tant que le mystère de la fraction troublante de $70 \%$ n'était pas éclairci.

Il convient de noter que, par rapport aux installations amont, l'étude d'une chambre d'équilibre en aval se complique du fait des inégalités dans la répartition des vitesses au débouché des aspirateurs des groupes; cette répartition peut d'ailleurs se modifier profondément avec la charge. Les données expérimentales de M. GarDEL ont été établies pour des écoulements très réguliers : elles fournissent une indication pré-

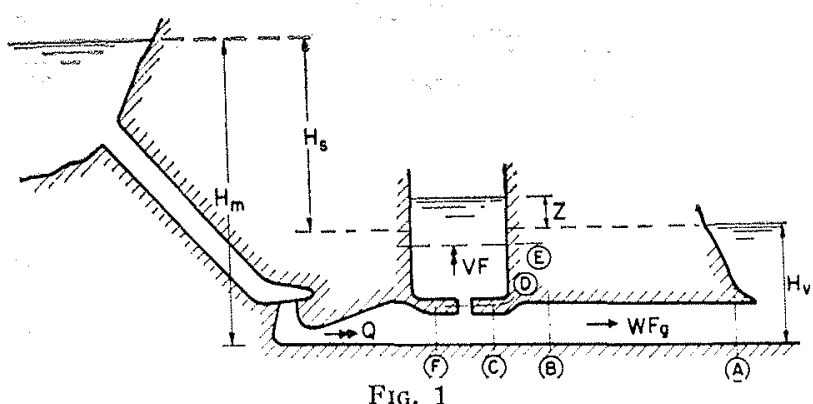

cieuse sur le comportement d'une cheminée aval communiquant avec la galerie de fuite dans le voisinage des aspirateurs, mais il va sans dire qu'une étude particulière sur modèle réduit, respectant pour chaque régime les données du constructeur des turbines, s'impose toutes les fois que le problème de la stabilité deva être spécialement examiné.

* Chambres d'Equilibre. - Analyse de quelques hypothèses usuelles; méthodes de calcul rapide. F. Rouge et Cie, Librairie de l'Université, Lausanne, 1956.
La présente note ne fait que compléter aussi succinctement que possible le travail de M. GARDEL dans un domaine particulier. Afin d'alléger le texte au maximum, nous suivons fidèlement l'enchaînement des équations et du texte du livre Chambres d'Equilibre, qui devra être consulté; en effet, nous ne pouvons reproduire les résultats expérimentaux ni même les raisonnements généraux de M. Gardel, et nous nous bornons, par conséquent (là où les développements sont analogues dans les deux cas), à formuler des équations sans les commenter.

\section{Notations}

Nous avons conservé la forme générale des équations de M. Gardel, en modifiant au besoin la définition de certains coefficients, distingués alors par l'adjonction de primes :

$$
\begin{aligned}
&+a_{1}^{\prime}= \frac{2 p_{0} h_{0}-1+e_{0}\left\{2 p_{0}+c_{1}^{\prime}+{ }_{+} c_{2}^{\prime} h_{0}+2 e_{0}+c_{3}\right\}}{h_{0}+e_{0}+c_{1}^{\prime}} \\
&{ }_{-} a_{1}^{\prime}= \frac{2 p_{0} h_{0}-1+e_{0}\left\{2 p_{0} c_{1}^{\prime}+{ }_{-} c_{2}^{\prime} h_{0}+2 e_{0}-c_{3}\right\}}{h_{0}+e_{0}-c_{1}^{\prime}} \\
&{ }_{+} a_{0}^{\prime}=\frac{h_{0}-2 p_{0}+2 e_{0} h_{\gamma}}{h_{0}+e_{0}+c_{1}^{\prime}} \\
&-a_{0}^{\prime}=\frac{h_{0}-2 p_{0}+2 e_{00} h_{\gamma}}{h_{0}+e_{0}-c_{1}^{\prime}} \\
&{ }_{m} a_{0}^{\prime}=\frac{1}{2}\left\{{ }_{+} a_{0}^{\prime}+\ldots a_{0}^{\prime}\right\}
\end{aligned}
$$

$c_{0} Z_{*}=$ pertes de charge dans la conduite forcée :

$$
\begin{array}{r}
{ }_{+} c_{1}^{\prime}=-{ }_{+} c_{2}=+m_{\beta}+2{ }_{0} h_{\beta} \\
\left.\quad \text { (valable pour } q_{\beta}>0\right)
\end{array}
$$

$$
\left.{ }_{-} c^{\prime}{ }_{1}=-c_{2}=-m_{\beta} \text { (valable pour } q_{\beta}<0\right) \text {; }
$$

d'où :

$$
{ }_{ \pm} c_{1}^{\prime}=-{ }_{ \pm} c_{2}= \pm m_{\beta}+2_{0} h_{\beta}(1 \text { ou } 0)
$$

De mème :

$$
\begin{aligned}
& \pm c_{2}^{\prime}=-{ }_{ \pm} c_{1}= \pm m_{\gamma \beta}+2{ }_{0} h_{\gamma \beta}(0 \text { ou }-1) ; \\
& \pm c_{3}=-{ }_{ \pm} m_{\beta} h_{\gamma \beta}+{ }_{ \pm} m_{\gamma \beta} h_{\beta} ; \\
& { }_{m} c_{1} \text {, etc., sont les valeurs moyennes de }{ }_{+} c_{1} \\
& \quad \text { et }-c_{1} \text {, etc.; } \\
& e_{0} Z_{*}=\mathrm{E}_{0}=\chi \frac{W^{2}{ }_{00}}{2 g} \text {, hauteur due à la vitesse } \\
& \text { à la base de la cheminée; }
\end{aligned}
$$




$$
\begin{array}{r} 
- \pm h_{\beta}\left\{\frac{\mathrm{V}_{\alpha}^{2}}{2 g} \text { ou } \frac{\mathrm{V}_{\gamma}^{2}}{2 g}\right\}=\mathrm{H}_{\alpha}-\mathrm{H}_{\beta}=\text { perte de } \\
\text { charge suivant le circuit } \alpha \beta \text { (voir } \\
\text { la fig. 2), la vitesse de référence } \\
\text { étant } \mathrm{V}_{\alpha} \text { si } q_{\beta}>0 \quad\left({ }_{+} h_{\beta}\right. \text { : écoule- } \\
\text { ment dirigé vers le haut dans la } \\
\text { branche } \beta \text { ), et } \mathrm{V}_{\gamma} \text { si } q_{\beta}<0 \quad\left({ }_{\beta} h_{\beta}\right. \text { : } \\
\text { écoulement dirigé vers le bas); }
\end{array}
$$

${ }_{\alpha} h_{\beta}$ : valeur de $h_{\beta}$ pour $q_{\beta}=0$;

$-{ }_{ \pm} h_{\gamma}\left(\frac{\mathrm{V}_{\alpha}{ }^{2}}{2 g}\right.$ ou $\left.\frac{\mathrm{V}_{\gamma}{ }^{2}}{2 g}\right)=\mathrm{H}_{\alpha}-\mathrm{H}_{\gamma}=$ perte de charge suivant le circuit $\alpha \gamma$ (voir cidessus);

${ }_{a} h_{\gamma}$ : valeur de $h_{\gamma}$ pour $q_{\beta}=0$;

$$
\begin{aligned}
& { }_{ \pm} h_{\gamma \beta}={ }_{ \pm} h_{\gamma}-{ }_{ \pm} h_{\beta} ; \\
& { }_{ \pm} m_{\beta}=\left(\frac{d{ }_{ \pm} h_{\alpha}}{d q_{\beta}}\right){ }_{q_{\beta}}=0
\end{aligned}
$$

Au voisinage de $q_{\beta}=0$, il vient approximativement :

$$
{ }_{ \pm} h_{\beta}={ }_{0} h_{\beta}+{ }_{ \pm} m_{\beta} q_{\beta} ;
$$

${ }_{ \pm} m_{\gamma \beta}$ : semblable à la définition précédente;

$p_{0} \frac{W_{0}^{2}}{2 g}=\left(\zeta+\zeta_{2}\right) \frac{W_{0}^{2}}{2 g}-{ }_{0} h_{\gamma} \frac{W_{c 0}^{2}}{2 g}=\mathrm{P}_{0}$,

pertes de charge dans la galerie, y compris la perte singulière au débouché aval;

$q \mathrm{Q}_{0}=\mathrm{Q}_{t}$, le débit instantané turbiné;

$q_{\beta}\left\{Q_{\alpha}\right.$ ou $\left.Q_{\gamma}\right\}=$ débit dans la branche $\beta$, $q_{\beta}$ étant rapporté au débit maximum des trois branches (voir la fig. 2);

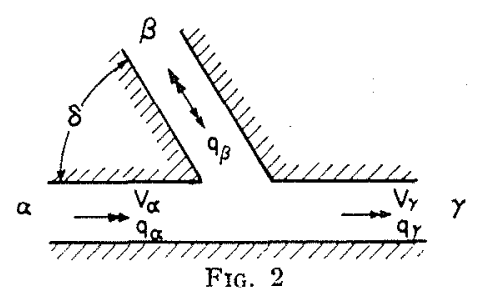

$t \mathrm{~T}_{g}=\mathrm{T}$, le temps;

${ }_{v} \mathrm{Q}_{0}=$ débit entrant dans la chambre;

$w \mathrm{Q}_{0}=$ débit de la galerie;

$x_{0} \mathrm{~F}_{t}=\mathrm{F}_{0}$, section de la chambre pour laquelle la stabilité est critique;

$z Z_{*}=Z$, la montée dans la chambre comptée au-dessus du niveau statique;
$\mathrm{E}_{f}=\chi \frac{\mathrm{W}_{f 0}{ }^{2}}{2 g}$

$\mathrm{E}_{y}=\frac{\mathrm{W}_{0^{2}}}{2 g}$, hauteur due à la vitesse dans la galerie;

$\mathrm{E}_{0}$ : voir sous $e_{0}$;

$\mathrm{F}=$ section de la chambre;

$\mathrm{F}_{c}=\mathrm{F}_{f}=$ section du dispositif Venturi à la base de la chambre;

$\mathrm{F}_{d}=$ section du piquage;

$\mathrm{F}_{g}=$ section de la galerie;

$\mathrm{F}_{0}$ : voir sous $x_{0}$;

$\mathrm{F}_{t}=\frac{\mathrm{W}_{0}{ }^{2}}{2 g} \cdot \frac{\mathrm{L} \mathrm{F}_{g}}{\mathrm{P}_{0} \mathrm{H}_{0}}$, section de Thoma calculée sans tenir compte de la hauteur due à la vitesse sous la chambre;

$\mathrm{H}_{a} \mathrm{H}_{b}$, etc. : Hauteurs de la ligne de charge aux points $A, B$, etc., comptées audessus d'un plan de référence quelconque;

$\mathrm{H}_{m}=$ niveau amont (compté au-dessus du même plan);

$\mathrm{H}_{0}=\mathrm{H}_{s}-\mathrm{P}_{0}$;

$\mathrm{H}_{s}=\mathrm{H}_{m}-\mathrm{H}_{v}$;

$\mathrm{H}_{3}=$ niveau aval;

$\mathrm{L}=$ longueur de la galerie;

$L^{\prime}=$ profondeur d'eau dans la cheminée;

$\mathrm{P}_{a} \mathrm{P}_{b}$ : hauteurs de la ligne piézométrique aux points $A, B$;

$\mathrm{P}_{b a}=$ pertes de charge sur le parcours

$$
\mathrm{BA}= \pm \zeta \frac{\mathrm{W}^{2}}{2 g} ;
$$

$P_{0}$ : voir sous $p_{0}$ plus haut;

$Q_{0}=$ débit turbiné en régime permanent;

$\mathrm{Q}_{t}=\mathrm{Q}_{0}+\Delta \mathrm{Q}$, débit turbiné instantané;

$\mathrm{S}=$ hauteur accélératrice;

$\mathrm{T}=$ temps;

$\mathrm{T}_{g}=\sqrt{\frac{\mathrm{LF}}{g \mathbf{F}_{g}}}$, temps «caractéristique 》de

$\mathrm{V}_{0}=\frac{\mathrm{Q}_{0}}{\mathrm{~F}} ;$ 
$\mathrm{V}=\frac{d Z}{d^{\prime} \mathrm{T}}=\frac{Z_{*}}{\mathrm{~T}_{g}} \frac{d z}{d t}$

$\mathrm{W}_{0}=\frac{\mathrm{Q}_{0}}{\mathrm{~F} g}$

$W=W_{0}+\Delta W$, vitesse instantanée dans la galerie;

$\mathrm{W}_{f}=\frac{\mathrm{Q}_{t}}{\mathrm{~F}_{f}}$

$\mathrm{W}_{c 0}=\mathrm{W}_{j 0}=\frac{\mathrm{Q}_{0}}{\mathrm{~F}_{c}}$

$\mathrm{Z}_{*}:=\mathrm{W}_{0} \sqrt{\frac{\mathrm{LF}}{g \mathrm{~F}_{g}}}$

$\alpha \beta \gamma$ : voir la figure 2 ;

$\delta=$ angle de piquage

$\zeta=$ coefficient de pertes de charge par frottement dans la galerie;

$\eta=$ rendement $\mathrm{du}$ groupe;

$\eta_{1 \text { ou } 2}=$ coefficient de perte de charge par étranglement à la base de la cheminée;

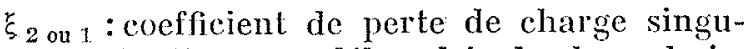
lière au débouché de la galerie. Pour $W>0$, M. GARDEL donne :

$$
0,4 \leqslant \zeta_{2} \leqslant 0,8
$$

$\chi \psi:$ coefficients de « cinéticité $»$, pouvant atteindre des valeurs élevées (de 2 à 3) au débouché d'une machine.

$\Delta$ désigne les écarts d'une variable par rapport à sa valeur moyenne.

\section{Mise en équations du problème des petites oscillations avec réglage automatique}

Nous faisons abstraction ici des pertes de charge dans la conduite forcée et des variations de rendement, quitte à revenir sur ces questions à la fin de notre exposé.

Il a été supposé que la puissance fournie par la turbine demeure constante dans le temps, grâce à l'intervention du régleur supposé parfait. Pour l'établissement de l'équation de puissance constante, il a été admis que le produit du débit et de la chute nette définie, selon les normes suisses, par la « différence de niveau des lignes d'énergie à l'amont et à l'aval de la turbine », est invariable; la définition' du rendement qui en résulte est conforme aux usages, toute autre façon de procéder ayant obligatoire- ment pour résultat de modifier la notion admise du rendement. Nous sommes amenés à faire cette remarque à la suite de la suggestion qui nous a été faite de prendre comme invariable le produit du débit et d'une chute égale à la différence entre la ligne d'énergie en amont et la pression en aval. Nous reviendrons sur ce point également à la fin de cette note.

La numérotation des équations s'identifie à celle de M. Garder, pour la commodité de la comparaison avec son livre.

Les équations de base sont les suivantes :

$$
\begin{aligned}
& \frac{\mathrm{L}}{g} \frac{d \mathrm{~W}}{d \mathrm{~T}}-\mathrm{S}=0 \\
& \mathrm{H}_{a}=\mathrm{P}_{a}+\frac{\mathrm{W}^{2}}{2 g} \\
& \mathrm{H}_{b}=\mathrm{H}_{c} \\
& \left.\mathrm{H}_{b}=\mathrm{H}_{a}+ \pm h_{\gamma \beta} \frac{\mathrm{W}^{2} \text { in } c}{2 g}\right\} \begin{array}{l}
\mathrm{W}_{f} \text { pour } \mathrm{V}>0 \\
\mathrm{~W}_{c} \text { pour } \mathrm{V}<0
\end{array}
\end{aligned}
$$

$\left.\mathrm{H}_{d}=\mathrm{H}_{c} \pm \eta_{1 . \text { ou } 2}\left(\frac{\mathrm{F}}{\mathrm{F}_{d}}\right)^{2} \frac{\mathrm{V}^{2}}{2 g}\right\}+\eta_{1}$ pour $\mathrm{V}>0$

$\mathrm{H}_{c}=\mathrm{H}_{v}+\mathrm{Z}+\frac{\mathrm{V}^{2}}{2 g}$

$\left.\mathrm{P}_{a}=\mathbf{H}_{v}-\left(1-\zeta_{2011}\right) \frac{\mathrm{W}^{2}}{2 g}\right\}-\xi_{2}$ pour $\mathrm{W}>0$

$\mathrm{P}_{b}=\mathrm{H}_{c}-\downarrow \frac{W^{2}}{2 g}$

$\mathrm{P}_{b a}= \pm \zeta \frac{\mathrm{W}^{2}}{2 g} \quad+$ pour $\mathrm{W}>0$

$$
\mathrm{S}=\mathrm{H}_{b}-\mathrm{H}_{a}-\mathrm{P}_{b a}
$$

Il s'ensuit que :

$$
\begin{gathered}
\mathrm{S}=Z=\left(\zeta+\xi_{2011}\right) \frac{\mathrm{W}^{2}}{2 g} \pm \eta_{1011}\left(\frac{\mathrm{F}}{\mathrm{F}_{t}}\right) \frac{\mathrm{V}^{2}}{2 g} \\
+ \pm h_{\gamma_{B}} \frac{\mathrm{W}^{2} \text { ou } c}{2 g}+\frac{\mathrm{V}^{2}}{2 g}
\end{gathered}
$$

D'autre part :

$$
\begin{gathered}
\mathrm{WF}_{g}=-\mathrm{VF}+\mathrm{Q}_{t} \\
\mathrm{~V}=\frac{d Z}{d \mathrm{~T}}
\end{gathered}
$$

Etant donné que :

$$
\mathrm{H}_{f}=\mathrm{H}_{c}- \pm h_{\gamma} \frac{\mathrm{W}_{f \circ}^{2} c}{2 g}
$$


la condition sur la puissance s'écrit :

$$
\begin{array}{r}
\mathrm{Q}_{t}\left[\mathrm{H}_{s}-\mathrm{Z} \mp \eta_{11 \ldots}\left(\frac{\mathrm{F}}{\mathrm{F}_{l}}\right)^{2} \frac{\mathrm{V}^{2}}{2 g}+{ }_{ \pm} h_{B} \frac{\mathrm{W}^{2}}{2 g}-\frac{\mathrm{V}^{2}}{2 g}\right] \\
=\mathrm{Q}_{0}\left[\mathrm{H}_{s}-\left(\zeta+\xi_{2}\right) \frac{\mathrm{W}_{0}^{2}}{2 g}+{ }_{0} h_{\gamma} \frac{\mathrm{W}_{c 0}^{2}}{2 g}\right]\left(150^{\prime}\right)
\end{array}
$$

\section{Transformation en équations aux écarts sans dimensions}

Nous trouvons les équations suivantes :

$$
\begin{aligned}
& \frac{d \Delta w}{d t}-\Delta z+2 p_{0} \Delta w-e_{0}\left\{_{0} h_{\gamma \beta}(2 \Delta q \text { ou } 2 \Delta w)\right. \\
&\left. + \pm m_{\gamma \beta} \Delta v\right\}=0\left(166^{\prime}\right) \\
& \Delta w=-\Delta v+\Delta q \\
& \Delta v=\frac{d \Delta z}{d t}
\end{aligned}
$$

$$
-\frac{\Delta z}{h_{0}}+\Delta q+\frac{e_{0}}{h_{0}}\left\{h_{\beta}(2 \Delta q \text { ou } 2 \Delta w)+ \pm m_{\beta} \Delta \nu\right\}=0
$$

De même que dans le texte de M. Gardrs, les doubles signes ou les alternatives se rapportent au sens positif ou négatif de l'écoulement dans la cheminée.

\section{Equation différentielle pour $\Delta z$}

Après élimination de $\Delta w$ et de $\Delta q$ des précé-

En pratique, on peut admettre que $x_{0}$ est fourni, à un degré d'approximation largement suffisant, par l'équation $\left(177^{\prime}\right)$ :

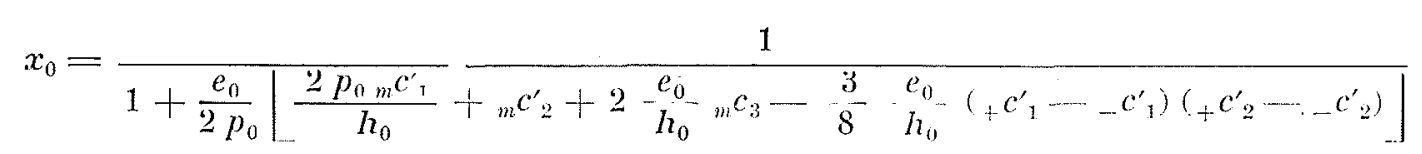

Pour des installations où $h_{0}$ est grand devant $p_{0}$ et $e_{0}$, il vient :

$$
\text { (A) } x_{0} \cong \frac{1}{1+\left(e_{0} / 2 p_{0}\right)_{m} c_{2}^{\prime}}=\frac{1}{1-\left(e_{0} / 2 p_{0}\right)_{m} c_{1}}
$$

L'imprécision que revêt la détermination des coefficients $c$ rend l'emploi de l'équation (177') d'une valeur assez douteuse, sauf dans des cas exceptionnels. Nous examinerons donc plus particulièrement dans ce qui suit l'application de l'équation (A).

Rappelons que le raisomnement simplifié auquel nous avons fait allusion dans l'introduction conduit à prendre :

$$
{ }_{m} c_{2}^{\prime}=--2
$$

La formule (A) devient alors :

$$
x_{0}=-\frac{1}{1-\left(e_{0} / p_{0}\right)}
$$

dentes, il vient $\left(168^{\prime}\right)$ :

$$
\begin{gathered}
\frac{d^{2} \Delta z}{d t^{2}}\left[h_{0}+e_{0} c_{1}^{\prime}\right]+\frac{d \Delta z}{d t} \\
{\left[2 p_{0} h_{0}-1+e_{0}\left(2 p_{0} c_{1}^{\prime}+c_{2}^{\prime} h_{0}+2 e_{0} c_{3}\right)\right]} \\
+\Delta z\left[h_{0}-2 p_{0}+2 e_{0} h_{\gamma}\right]=0
\end{gathered}
$$

Cette équation fondamentale ne diffère de l'équation (168) de M. Garder que par les coefficients $c_{1}^{\prime}$ et $c_{2}^{\prime}$ (remplaçant $c_{1}$ et $c_{2}$ ).

Nous pouvons, par conséquent, profiter de son analyse, donnée aux pages 92 et suivantes de son ouvrage, pour déduire directement la valeur de $x_{0}$ dans le cas qui nous intéresse.

\section{Section critique d'une chambre aval, compte tenu de l'énergie cinétique au droit de l'ouvrage.}

La valeur exacte de la section critique est fournie par la solution de l'équation :

$$
+\frac{a_{1}^{\prime}}{-a_{1}^{\prime}} \sqrt{\frac{-a_{0}^{\prime}}{+a_{0}^{\prime}}}=-1
$$

Pour retrouver ce résultat en partant de l'équation $\left(177^{\prime}\right)$, il faut faire les hypothèses que voici :

$1^{\circ}$ Ne considérer que le cas $\Delta z^{\prime}>0\left(q_{\beta}>0\right)$;

2" Prendre :

$$
\begin{aligned}
{ }_{0} h_{\beta} & =-1 \\
{ }_{0} h_{\gamma \beta} & =+1 \\
{ }_{0} h_{\gamma} & =0
\end{aligned}
$$

3" Prendre :

$$
\begin{array}{r}
{ }_{+} m_{\beta}=+2 \\
+m_{\gamma \beta}=-2
\end{array}
$$

On trouve alors que :

$$
\begin{aligned}
& +c_{+}^{\prime}=0 \\
& { }_{+} c_{1}^{\prime}=-2 \\
& { }_{+} c_{3}=0
\end{aligned}
$$

et, d'après $1^{\circ}$, ces valeurs s'identifient à celles de ${ }_{m} c_{1}^{\prime},{ }_{m} c_{2}^{\prime},{ }_{m} c_{3}^{\prime}$. 
M. Garder, qui trouve des résultats analogues, fait remarquer que la source d'erreur principale réside dans le choix des valeurs de $m$.

\section{Comparaison de la formule exacte (A) et de la formule classique (B)}

Cette comparaison fait intervenir uniquement la valeur de ${ }_{m} c_{1}$.

En partant de considérations d'ordre quasi théorique, M. Garder a dressé, à la page 94 de son livre, des abaques pour les trois coefficients ${ }_{m} c_{1},{ }_{m} c_{2},{ }_{m} c_{3}$. Nous avons préféré reprendre ses résultats expérimentaux bruts pour évaluer ${ }_{m} c_{1}$, en partie parce que cette manière de faire nous a permis de tirer profit des mesures faites à Iowa (voir J. McNown, « Mechanics of Manifold Flow », Separate No. 258, Proceedings American Society of Civil Engineers, août 1953), et surtout parce que la théorie dont il s'agit n'admet pas les valeurs « paradoxales » de ${ }_{0} h_{\gamma}$, valeurs positives dont M. McNown justifie l'existence; or, M. Gardel a effectivement mesuré de telles valeurs, et ses courbes théoriques s'en écartent nettement.

En opérant de la sorte, pour $\Phi=1$, nous avons trouvé :

$$
\begin{aligned}
{ }_{m} c_{1} & =-0,4 \text { à } 0,5 \text { pour } & \grave{o} & =45^{\circ} \\
& =-0,4 \text { environ } & & =60^{\circ} \\
& =-0,3 & & =75^{\circ} \\
& =-0,3 \text { environ } & & =90^{\circ} \\
& =-0,10 & & =105^{\circ} \\
& =-0,17 & & =120^{\circ} \\
& =-0,27 & & =135^{\circ}
\end{aligned}
$$

Revenant aux formules, on voit que, contrairement aux conclusions généralement admises jusqu'ici,

1"L'énergie cinétique jouerait, dans la plupart des cas, un rôle bienfaisant à l'égard de la stabilité, car $m_{1} c_{1}$ serait en général négatif;

$2^{\circ}$ L'amélioration serait surtout sensible pour un piquage forfement incliné, du genre éjecteur ( $\delta$ petit) et de faible section ( $\Phi$ petit). Or, un tel piquage a apparemment pour effet d'abaisser le niveau dans la chambre et, selon les idées conmunément admises qui font dépendre la stabilité de la dénivellation en régime uniforme entre chambre et restitution $\left(^{*}\right)$, de compromettre le bon fonctionnement de l'installation. S'il n'en est rien, en fait, il faut bien dire que, pour les piquages à bord arrondi essayés par M. GARDEL, le niveau dans la chambre s'établit très près de la ligne piézométrique, quelle que soit la valeur de $\delta$.

\section{Influence d'une autre définition de la chute}

Il a été question, dans l'Introduction, d'une définition faisant intervenir la hauteur piézométrique à l'aval, au lieu de la hauteur de la ligne de charge, ce qui revient à ajouter au còté gauche de l'équation (150') un terme :

$$
\mathrm{Q}_{\ldots} . \% \frac{\mathrm{W}_{j}^{2}}{2 g}=\mathrm{Q}_{t} \mathrm{E}_{f}\left\{1+2 \frac{\Delta \mathrm{O}}{\mathrm{Q}_{0}}\right\}
$$

el à modifier en conséquence la définition de $\mathrm{H}_{0}$ figurant du côté droit.

On démontre que, dans nos formules, les coefficients dépendant :

$1^{\circ}$ de $\gamma \beta$, demeurent inchangés,

$2^{\circ}$ de $\beta$, se trouvent augmentés de :

$$
\chi \frac{\mathrm{E}_{f}}{\mathrm{E}_{0}}=\% \text { si } \mathrm{E}_{f}=\mathrm{E}_{0}
$$

$3^{\circ}$ de $\gamma$, doivent être diminués de la même quantité.

Ainsi, dans l'équation fondamentale $\left(177^{\prime}\right)$ : ${ }_{m} c_{1}^{\prime}$ se transforme en ${ }_{m} c_{1}^{\prime}+2 \% ;{ }_{m} c_{2}^{\prime}$ reste inchangé et ${ }_{m} c_{3}$ devient ${ }_{m} c_{3}+\chi_{m} c_{2}^{\prime}$.

\section{Influence des pertes de charge dans la conduite forcée}

D'une façon analogue, on trouve que, pour tenir compte des pertes de charge dans la conduite forcée, il suffit de, remplacer, dans la formule $\left(177^{\prime}\right)$ :

(D) et :

$$
{ }_{m} c_{1}^{\prime} \text { par }{ }_{m} c_{1}^{\prime}-2 c_{0}
$$

$$
{ }_{m} c_{3} \text { par }{ }_{m} c_{3}-c_{0 m} c_{2}
$$

${ }_{m} c_{2}^{\prime}$ restant inchangé.

La correction introduite dans le calcul par la définition innovatrice de la chute dont il vient d'être question s'identifie, au signe près, à la correction faite pour tenir compte de la perte de charge $c_{0}$.

Dans ce dernier cas, on retrouvera la condition de stabilité «classique»:

$$
\left\{\frac{1}{2 c_{0}-h_{0}}+2\left(p_{0}-e_{0}\right)\right\}=0
$$

correspondant à l'équation (115) de M. Garbes, en employant les valeurs inexactes $(C)$ des coefficients ${ }_{m} c^{\prime}$.

\section{Influence $\mathrm{du}$ rendement}

Le calcul exact, correspondant aux relations (D), permettant de tenir compte de $c_{\phi}$, reste à faire. En nous replaçant dans les hypothèses du calcul « classique », nous pouvons reprendre la formule (118) établie par M. Gardel pour un système cheminée-galerie en amont, l'influence de $\eta$, comme celle de $c_{0}$, étant identique dans les deux cas. Vu l'imprécision des coefficients ${ }_{m} c^{\prime}$, cette façon de procéder est peut-être suffisante.

Il convient de faire remarquer que, si l'on

(*) C'est l'origine du terme $\left(p_{v}-e_{i}\right)$ figurant au dénominateur de l'expression (B). 
adopte la définition « innovatrice» de la chute, les courbes de rendement s'en trouveront affectées. La nouvelle courbe donnant $\eta_{1}$ en fonction de la puissance à chute constante, s'écartera progressivement de la courbe établie selon les normes habituelles, et il est logique de penser que, de ce fait, le bénéfice pour la stabilité résultant de l'introduction de $\%$ dans nos formules serait annulé en fin de compte.

\section{Influence de l'accélération de l'eau dans la cheminée}

Il nous a été suggéré que les résultats si paradoxaux de notre étude résultaient de l'oubli de cette influence, habituellement négligée et dont les essais de M. Garder, effectués en régime permanent, ne pouvaient tenir compte.

Il s'agirait de remplacer (141') par l'équation plus exacte :

$$
\mathrm{H}_{e}=\mathrm{H}_{v}+\mathrm{Z}+\frac{V^{2}}{2 g}+\frac{\mathrm{L}^{\prime}}{g} \frac{d \mathrm{Y}^{r}}{\alpha \mathrm{T}}
$$

Dans les équations qui suivent celle-ci, il faudrait ajouter à $Z$ un terme :

$$
\frac{\mathrm{L}^{\prime}}{g} \frac{d \mathrm{~V}}{d \mathrm{~T}}
$$

ou, encore, à $z$, un terme :

$$
\left(\frac{\mathrm{L}^{\prime}}{\mathrm{L}} \frac{\mathrm{F}_{q}}{\mathrm{~F}}\right) \frac{d^{2} z}{d t^{2}}
$$

Or, nous examinons le cas limite d'oscillations entretenues; $z$ varie alor's de façon quasi sinusoïdale, avec :

$$
\frac{d^{2} z}{d t^{2}} \cong-{ }_{m} a_{0}^{\prime} z
$$

proportionnel à $z$. L'équation (168') reste inchangée, car tous les termes sont multipliés par un facteur :

$$
1-\left\{\frac{\mathrm{L}}{\mathrm{L}} \cdot \frac{\mathrm{F}_{g}}{\mathrm{~F}}\right\rangle_{m^{\prime}} a_{0}^{\prime}
$$

qui disparaît; l'expression $\left(177^{\prime}\right)$ n'en est pas modifiée.

Il est vrai que, pour une cheminée instable du genre que nous considérons plus spécialement ( $\mathrm{L}$ petit), le facteur (E) pourrait modifier sensiblement l'amplitude des oscillations entretenues d'amplitude finie, de même que les grands mouvements provoqués par des changements de régime. It faudrait alors cependant reprendre le problème entièrement.

\section{CONCLUSION}

Si l'on ne considère que l'influence de la hauteur due à la vitesse à la base d'une cheminée aval sur la stabilité de cet ouvrage, on peut en première approximation retenir une formule :

$$
x_{0}=\frac{1}{1+(1 / 2) \chi_{m} c_{2}^{\prime}\left(\mathbf{E}_{g} / p_{0}\right)}
$$

Le facteur $(1 / 2) \chi_{m} c_{2}^{\prime}$ semble pouvoir atteindre des valeurs positives assez notables, alors que la théorie « classique » admise jusqu'ici n'admettait que la valeur - 1. Ainsi, l'éneroie cinétique $\mathrm{E}_{0}=\% \mathrm{E}_{g}$ pourrait bien contribuer en fin de compte (et selon les piquages adoptés) à rendre une cheminée aval stable, ce qui est tout à fait inaltendu.

En raison toutefois des conditions particulieres régnant au débouché d'un aspirateur, il faudrait effectuer des études approfondies de cette question sur modèle réduit. En effet, la répartition des vitesses et la valeur de $\%$ varient, non seulement d'une machine à une autre, mais encore en fonction de la charge pour une même turbine. De tels essais auraient pour but pratique de mettre au point des piquages favorisant la stabilité dans les différents cas de fonctionnement.

Une étude expérimentale de chaque installation particulière sur modèle réduit en similitude dynamique (c'est-à-dire en reproduisant des montées et descentes du plan d'eau qu'on aura dans la nature) est motivée d'ailleurs:

- Par la présence de la surface libre, ce qui peut conduire à la formation de vortex ou de " champignons » impossible à représenter dans une installation à écoulement continu;

- Par la nécessité de tenir compte des rouleaux qui seront engendrés dans la masse d'eau dans la cheminée par les courants entrant dans celle-ci en particulier lors des montées; la création et l'amortissement de ces rouleaux ne peuvent être reproduits dans une installation à écoulement continu. Nous pensons toutefois qu'ils sont susceptibles de modifier les coefficients établis par M. GARDEL.

Ces dernières remarques sont évidemment valables aussi bien pour des cheminées en amont que pour celles en aval.

Les autres facteurs, $\eta, c_{0}$ etc, pouvant influer sur la stabilité, interviennent, d'après le calcul "classique » et non rigoureux, de la même facon que dans le cas d'une cheminée en amont. Un calcul plus précis, que nous avons d'ailleurs effectué pour le facteur $c_{0}$, reste possible, mais il se peut bien que la précision atteinte de la sorte soit en partie illusoire, en raison des difficultés éprouvées dans la détermination des coefficients expérimentaux. 